\title{
A REVIEW: SIMULTANEOUS LOCALIZATION AND MAPPING IN APPLICATION TO AUTONOMOUS ROBOT
}

Agunbiade OY and Zuva T

Vaal University of Technology,

Andries Potgieter Blvd Vanderbijlpark 1900, South Africa.

email:agunbiadeolusanya@yahoo.com/agunbiadeOY@tut.ac.za

\begin{abstract}
s
The important characteristic that could assist in autonomous navigation is the ability of a mobile robot to concurrently construct a map for an unknown environment and localize itself within the same environment. This computational problem is known as Simultaneous Localization and Mapping (SLAM). In literature, researchers have studied this approach extensively and have proposed a lot of improvement towards it. More so, we are experiencing a steady transition of this technology to industries. However, there are still setbacks limiting the full acceptance of this technology even though the research had been conducted over the last 30 years. Thus, to determine the problems facing SLAM, this paper conducted a review on various foundation and recent SLAM algorithms. Challenges and open issues alongside the research direction for this area were discussed. However, towards addressing the problem discussed, a novel SLAM technique will be proposed.
\end{abstract}

Keyword: Autonomous robot, illumination variance, kidnap robot, dynamic environment, navigation, simultaneous localization and mapping

\section{INTRODUCTION}

Simultaneous Localization and mapping (SLAM) is an important problem that has been broadly researched in robotics. Its contribution towards autonomous robot navigation has attracted researchers towards focusing on this area (Fuentes-Pacheco et al., 2015, Zunino and Christensen, 2001). In the past, various techniques for addressing simultaneous localization and mapping has been proposed with remarkable achievements. Research had been conducted based on several types of sensors because of their advantage to one another; also sensor selection can be as a result of the type of technique they are proposing to solve the SLAM problem (Steckel and Peremans, 2013). In (Eliazar and Parr, 2003), they proposed to address the SLAM problem in an environment without pre-determined landmarks using laser sensors. The Particle filter algorithm was deployed on their system and had produced a highly detailed map for an office environment. Laser sensors apart from being expensive could produce a wrong measurement when encountering shiny or black objects that do not reflect light and this could affect robot localization in an environment (Agunbiade et al., 2014). In (Zunino and Christensen, 2001) they proposed the use of the EKF filter algorithm to process the information obtained by the sonar sensors attached to the robot. The sonar sensor was proposed because of its low cost and low computational complexity for retrieving information from the environment. In the work of (Steckel and Peremans, 2013), they condemn the use of sonar sensors because of its inability to provide fine-grained information from sound. Instead, they proposed bio-sonar. This was employed due to high intelligent interaction capability towards a complex environment and its ability to extract more information from the echoes than sonar. BatSLAM algorithm was proposed to analyse the information acquired by the bio-sonar, but from their experiment, the system limitation occurs if it encounters a larger complex environment because 
echoes arriving from different directions are delayed. Trying to analyse them produced an invalid cue which makes the system fail to navigate correctly (Hiryu et al., 2010). In the work of (Irie et al., 2012), they proposed a vision-based SLAM, because the camera was able to acquire more information from the environment than other sensors which could improve robot navigation (Zehang et al., 2006). Thus, being aware of the issues of environmental noise with vision-based system, they tend to address the issue of shadow. They proposed the use of two-dimensional occupancy grid maps produced from 3-D point clouds obtained by a stereo camera, they also introduced an extracted salient line segments from the ground into the grid map. On the grid map, robot pose estimation was attained by employing particle filters. In this technique, the grid maps were not affected by shadow and lighting conditions, but under severe illumination condition, it is impossible to extract the salient line segment which resulted to a failed SLAM technique. However, the issue of illumination variance support while some researchers still prefer the use of active sensors to acquire data from the environment (Thamrin et al., 2012). Furthermore, for reliable and accurate measurement of the environment, the work of (Castellanos et al., 2001) propose the use of multiple sensors to attain an impressive result so that one sensor can take advantage over the weakness of another. But the major limitation is high computational complexity when combining too much data from multiple sensors. Hence, irrespective of the sensor employed, they all have their limitations. However, sensors are not the only contributor to SLAM failure, algorithm employed to address the SLAM problem also have their limitations and they will be discussed in the next section. In this paper, Section 2 and 3 discuss the foundation and recent SLAM algorithm while section 4 discusses the challenges, open issues and research direction.

\section{FOUNDATIONAL SIMULTANEOUS LOCALIZATION AND MAPPING ALGORITHMS}

In an attempt to develop an efficient and effective technique that can address SLAM problem, sensors play an important role of acquiring data from the environment (Zehang et al., 2006) but localization and mapping techniques are not limited to this operation. There are several procedure that still need to be implemented, for instance, the analysis of the data captured assist in mapping building and localization, this can be attained by using SLAM algorithm (Chen, 2013). In the literature, several foundation SLAM algorithms has been proposed with outstanding result but they are all confronted with various challenges and issues (Hadji et al., 2014). In this section, some of these algorithms will be discussed together with their limitation and advantages.

\subsection{Extended Kalman Filter}

In the review involving Extended Kalman Filter (EKF), it is important to mention the Kalman filter because it is the foundation for EKF and some algorithms like Extended Information Filter (EIF), nonlinear least-square etc. (Hadji et al., 2014). Researchers over the years have employed Kalman filter as an algorithm to estimate dynamic linear systems with Gaussian noise (Chen, 2013). Kalman filter represent a state vector $\left(\mu_{t}\right)$ given in equation (1).

$$
\mu_{t}=\left(S_{t}, l_{1}, l_{2} \ldots \ldots \ldots, l_{N}\right)
$$


It is formulated by estimating the landmark $(l)$ where $\mathrm{N}$ represents numbers of map landmark, the current pose $\left(S_{t}\right)$, as well as a covariance matrix $\left(\sum_{t}\right)$ which signifies the covariance between the state variance also a measure of confidence. However, the issue of non-linearity in the robot model is the limitation of the Kalman filter algorithm and trying to address this issue led to Extended Kalman filter (Hadji et al., 2014).

Extended Kalman Filter (EKF) is an upgraded version of a Kalman filter that can address non-linear model (Chen, 2013). The linearization of non-linear model can be solved by many methods but in EKF, a technique called first order Taylor expansion is employed to address this issue. At each time ( $\mathrm{t}$ ), it linearize the measurement and motion model using the current state to estimate for a new update (Chen, 2013). The filter procedure is attained with two steps given in section 2.1.1 and 2.1.2.

\subsubsection{The Time Update stage}

At this stage, the filter computes the covariance matrix $\hat{\Sigma}_{t}$ and the predicated state $\hat{\mu}_{t}$ at time (t). Expressions is given in equations (2) and (3).

$$
\begin{aligned}
& \hat{\mu_{t}}=f\left(\mu_{t-1}, u_{t}\right) \\
& \hat{\Sigma}_{t}=A_{t} \sum_{t-1} A_{t}^{T}+G \wedge_{u} G^{T}
\end{aligned}
$$

where $A_{t}$ signifies the Jacobian of the motion model $f$ as related to the robot pose $S_{t}$ that is evaluated at $\mu_{t}, u_{t}$ represent the robot control, $\wedge_{u}$ signifies the covariance matrix related to this stage and $G$ represent a projection matrix.

\subsubsection{The measurement update stage}

This stage plays a significant role to address the problem of data association $(c)$ and generate the new updated measurement for $\mu_{t}$ and $\sum_{t}$ using the current state of the previous stage. They are computed by estimating first, the Kalman gains as given in equations (4)-(6).

$$
\begin{aligned}
K_{t} & =\hat{\sum}_{t} C_{t}^{T}\left(C_{t} \hat{\sum}_{t} C_{t}^{T}+\wedge_{Z}\right)^{-1} \\
\mu_{t} & =\hat{\mu}_{t}+K_{t}\left(z_{t}-h\left(\hat{\mu}_{t}, c\right)\right) \\
\sum_{t} & =\left(I-K_{t} C_{t}\right) \hat{\sum}_{t}
\end{aligned}
$$

Where $I$ represent identity matrix, $K_{t}$ represents the Kalman gain, $C_{t}$ represent the Jacobean of the measurement model $h$ in relation to every detected land marks estimated at $\hat{\mu}$ and the pose of the 
robot, $\wedge_{z}$ signifies the covariance matrix as related to this stage and $z_{t}$ represent the sensor measurement (Chen, 2013).

The above listed two steps are unique to EKF and can be use to instantiate the online SLAM given a condition of Gaussian model. The EKF is a popular algorithm because of the ability to overcome the problem of Kalman filter, but the computational cost of the algorithm is high (Dissanayake et al., 2011).

\subsection{Sparse Extended Information Filter}

Considering the limitation of high computational cost of EKF, this shifted researchers attention to a more advance filter known as Extended Information Filter (EIF). In EIF high computational cost was reduced by avoiding the computation of the Gaussian posterior in terms of the mean $\mu$ and the covariance matrix $\sum$ instead, the filter employs the information form of the posterior, which uses the information matrix $H$ and information vector $b$ (Chen, 2013). The parameterization is given below in equation (7) and (8)

$$
\begin{aligned}
& H=\sum^{-1} \\
& b=\mu^{T} H
\end{aligned}
$$

The EIF operational function is related to EKF, but the time update and measurement update stage are parallel to that of EKF, the equation 5 and 6 of the Extended Kalman Filter are transformed in EIF using the generated parametric value of information vector and information matrix (Chen, 2013). The parametric substitution for these stages are given in equations (9) - (12).

\subsubsection{The Time Update stage}

$$
\begin{aligned}
& \hat{H}_{t}=\left[\left(I+A_{t}\right) H_{t-1}^{-1}\left(I+A_{t}\right)^{T}+S \wedge_{u} S^{T}\right]^{-1} \\
& \hat{b}=\left(b_{t-1} H_{t-1}^{-1}+f\left(\mu_{t-1}, u_{t}\right)^{T}\right) \hat{H}_{t}
\end{aligned}
$$

where $I$ represent identity matrix, $A_{t}$ signifies the Jacobean of the motion model $f$ estimated at $\mu_{t}$, the projected matrix is signified as $S$. At this stage, the updated time estimated is managed to its best because of the mean $\left(\mu_{t}\right)$ recovery and the inversion of a dense $H_{t}$. However, constant time updates can be attained if $H_{t}$ has a sparse characteristic and $\mu_{t}$ is available for all landmarks and robot poses, this is attained at the next stage (Chen, 2013).

\subsubsection{The measurement updated stage}

$$
\begin{aligned}
& H_{t}=\hat{H}_{t}+C_{t} \wedge_{z}^{-1} C_{t}^{T} \\
& b_{t}=\hat{b}_{t}+\left(z_{t}-\hat{z}_{t}+C_{t}^{T} \mu_{t}\right)^{T} \wedge_{z}^{-1} C_{t}^{T}
\end{aligned}
$$


where $z_{t}-\hat{z}_{t}$ represent the difference between updated and current sensor measurement, $C_{t}$ signifies the Jacobean of the measurement model estimated at $\mu_{t}$. At this stage, it requires only constant time summation taking into consideration that $C$ is sparse with non-zero values for the observe landmarks and pose in the measurement (Chen, 2013). Given this condition for every step of a matrix, non-zero values will only be associated to $\hat{H}_{t}$. EIF is regarded as an approximation approach with an improved processing speed better than EKF because of low computational complexity. However, as a result of approximation, the issue of inconsistency has not been resolved (Dissanayake et al., 2011).

\subsection{Rao-Blackwellized Particle Filter}

In the work of Murphy, Rao-Blackwellized particle filter is implemented to estimate the joint posterior $\left(p\left(S_{1: t}, m \mid Z_{1: t}, U_{1: t-1}\right)\right)$ of a map $(m)$ and the robot trajectory $\left(S_{1: t}=S_{1}, \ldots \ldots \ldots, S_{t}\right)$. The estimation is carried out given the odometry measurement $\left(U_{1: t-1}=U_{1}, \ldots \ldots \ldots, U_{t-1}\right)$ and the observation measurement $\left(Z_{1: t}=Z_{1}, \ldots \ldots . . ., Z_{t}\right)$ of the robot. Using the information provided above, the Rao-Blackweilized particle filter used the factorization given in equation (13) to represent SLAM (Wurm et al., 2003).

$$
p\left(S_{1: t}, m \mid Z_{1: t}, U_{1: t-1}\right)=p\left(m \mid S_{1: t}, Z_{1: t}\right) \cdot p\left(S_{1: t} \mid Z_{1: t}, U_{1: t-1}\right)
$$

In the factorization procedure, the trajectory of the robot is first estimated, then the map given that same trajectory computed. This is mandatory because the map creation rely on the estimated robot pose [22]. The Rao-Blackwelized particle filter offers efficient computational cost with improved processing speed. Its representation in equation (13) can be formulated efficiently to address SLAM since the posterior of the map $p\left(m \mid S_{1: t}, Z_{1: t}\right)$ will be estimated by employing the technique of mapping with known poses given that $S_{1: t}$ and $Z_{1: t}$ are known (Wurm et al., 2003). In computing the posterior $p\left(S_{1: t} \mid Z_{1: t}, U_{1: t-1}\right)$ for potential trajectories, particle filter may be employed. Since every particle corresponds to a potential robot trajectory and individual maps are connected with each sample. Therefore, maps will be created using the observation and corresponding particle relating to the trajectory. This procedure allows the robot to learn models of their environment and estimate successfully their trajectory (Wurm et al., 2003). Thus, the particle filters effectiveness and complexity rely heavily on the number of particles. The increase in the number of particles might improve its effectiveness, but at a price of high computational cost. Otherwise, the effectiveness can be minimized with low computational cost. However, estimating an optimal number of particle required is often difficult to attain (Montemerlo and Thrun, 2003).

In the literature, the above mentioned foundation SLAM algorithms are very common [9] but not limited to these three algorithms, others such as, Unscented Kalman filter (UKF) and Compressed Extended Kalman Filter (CEKF) exist (Hadji et al., 2014). The algorithms discussed above are the basis of operation for other algorithms (Chen, 2013). Irrespective of the algorithm proposed, they all have their advantages and limitations, and these shows that more research still need to be done in this area. However, researchers do not only rely on these algorithms but they tend to introduce more components that can improve SLAM performance, some of their methods will be presented in the next section. 


\section{RECENT PROPOSED SLAM ALGORITHMS}

The simultaneous localization and mapping is an important problem to consider as far as autonomous guidance is concerned (Fuentes-Pacheco et al., 2015). Thus, successful navigation of a robot given in an unknown environment require continuous updating of map while simultaneously estimating its position in its environment. In literature, researchers have presented recent technique to overcome the challenges of the foundational SLAM algorithm (Khairuddin et al., 2015). This section will discuss some of these techniques, their challenges and issue they encountered during their implementation. Furthermore, this section will disclose recent issue because reviews are only conducted on recent techniques only.

In the work of (Agha-mohammadi et al., 2015), they propose to replan at every time $(k)$ when there is a probability distribution update on the state of the autonomous robot, this technique is referred to as: Simultaneous localization and Planning. The algorithm employed to carry out this task is known as Partially Observable Markov Decision Process (POMDP). This algorithm was proposed because of its ability to cope with uncertainties and changes. The idea employed in motion planning under uncertainty is to identify a policy $\left(\pi_{k}\right)$ at each time $(k)$ that generate control state $\left(U_{k}\right)$ using the available information of the robot. Thus, there are other important terms that needs to be defined to achieve this goals. Given a robot in an unknown environment whose state is represented by $x_{k}$ at time step $(k)$, the motion noise and control state at time $(k)$ is represented by $W_{k}$ and $U_{k}$ respectively. The state evolution model can now be formulated using equation (14)

$$
x_{k+1}=f\left(x_{x}, U_{k}, W_{k}\right)
$$

However, in any partial observable system, the sensor vector measurement at very time $k$ represented as $Z_{k}$ plays an important role of providing observation measurement. The expression for $Z_{k}$ is given in equation (15).

$$
Z_{k}=h\left(x_{k}, V_{k}\right)
$$

where $V_{k}$ denotes sensing noise.

On this note, the data available for deriving decision at each time $k$ is the history of controls and observation as expressed in equation (16), the conditional probability distribution for the overall possible robot state is given in equation (17)

$$
\begin{aligned}
& \mathrm{H}_{k}=\left(Z_{0: K}, U_{0: k-1}\right)=\left(Z_{0}, Z_{1}, \ldots \ldots . ., Z_{k}, U_{0}, \ldots \ldots \ldots, U_{k-1}\right) \\
& b_{k}=p\left(x_{k} \mid H_{k}\right)
\end{aligned}
$$


The $b_{k}$ generated in equation (17) is also referred to as information state or belief that compressed the data $\mathrm{H}_{k}$ and can be recursively computed using the last state and current observation as expressed in equation (18)

$$
b_{k+1}=\alpha p\left(Z_{k+1} \mid x_{k+1}\right) \int_{X} p\left(x_{k+1} \mid x_{k}, U_{k}\right) b_{k} d x_{k}
$$

where $\alpha$ represent the normalization constant and the $U_{k}$ can be generated based on the information state using a policy $\pi_{k}$ as expressed in equation (19)

$$
U_{k}=\pi_{k}\left(b_{k}\right)
$$

the $\pi_{k}$ represent the solution of a POMDP over a continuous observation space with a limitation that is intractable. In addressing this issue, Feedback based Information Road Map (FIRM) was proposed to minimize the intractable problem to a tractable POMDP by generating a representative graph in the information state space.

Given a FIRM graph with controller, policy $\pi^{g}$ can be extracted by mapping graph nodes $(v)$ to the edge $(m)$ as expressed in equation (20)

$$
\pi^{g}: v \rightarrow m
$$

Thus, the set of all graph planar $\left(\Pi^{g}\right)$ generated in an information state space allows POMDP to be tractable on the FIRM graph as expressed in equation (21)

$$
\pi^{g^{*}}=\underset{\Pi_{g}}{\arg \min } \mathrm{E} \sum_{n=o}^{\infty} C^{g}\left(B_{n}, \pi^{g}\left(B_{n}\right)\right)
$$

where visited $\mathrm{n}^{\text {th }}$ node is represented by $B_{n} ., C^{g}\left(B_{n}, \pi^{g}\left(B_{n}\right)\right)$ signifies the cost function. Experimental performance attained is impressive, but the limitation is inability to cope with dynamic environment. In their future work, they want to propose a frame work that can learn and model changes using prior knowledge of object motion.

The technique proposed in the study of (Tian and Ma, 2016) to address the problem of SLAM is known as double guarantee kidnapping detection (DGKD). Comparing the DGKD with other SLAM technique, 2 new processes were introduced to double check and guarantee detection and the type of detection. In carrying out this task, a threshold for the metric on real-time conditions was determined and introduced to avoid misjudgment. This increases the reliability and present deformation of information. Figure 1 represents the DGKD work flow. 


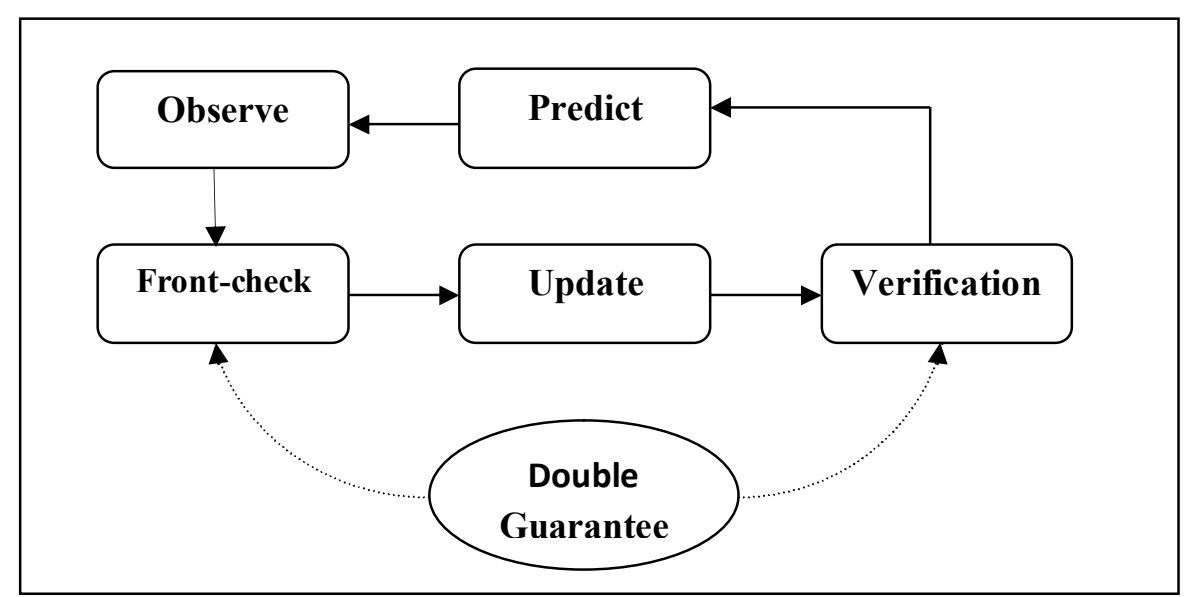

Figure 1: The overall workflow of the DGKD model (Tian and Ma, 2016)

However, DGKD limitations is the inability to cope with relatively large-scale environment and to increase its adaptability in large scale environment, the probability of features position and robot pose was combined to DGKD to form a new technique known as Probabilistic double guarantee kidnapping detection (PDGKD). In PDGKD, giving a robot state $\left(X_{r}\right)$ expressed in equation (22) and the state of feature $\left(X_{m}\right)$ given in equation (23).

$$
X_{r}=\left(x_{r}, y_{r}, \phi_{r}\right)^{T}
$$

where $\left(x_{r}, y_{r}\right)$ signifies position while $\phi_{r}$ denote frame orientation of the robot,

$$
X_{m}=\left(X_{m_{1}}^{T}, X_{m_{2}}^{T}, \ldots \ldots . . .\right)^{T}
$$

while $X_{m i}$ signifies the position of the feature $(i)$ in the global coordinate as expressed in equation (24).

$$
X_{m i}=\left[\begin{array}{cc}
\cos \phi_{r} & -\sin \phi_{r} \\
\sin \phi_{r} & -\cos \phi_{r}
\end{array}\right]\left[\begin{array}{c}
{ }^{L} x_{i} \\
{ }^{L} y_{i}
\end{array}\right]+\left[\begin{array}{l}
x_{r} \\
y_{r}
\end{array}\right]
$$

${ }^{L} x_{i},{ }^{L} y_{i}$ denotes the position of feature $i$ referred to the local coordinates frame attached on the robot.

Hence, the derived state vector combines both features state $\left(X_{m}\right)$ and robot state $\left(X_{r}\right)$ has expressed in equation (25) to address the DGKD SLAM problem.

$$
X=\left(X_{r}^{T}, X_{m}^{T}\right)^{T}
$$


In PDGKD, the operations are not only limited to the above mentioned process, there are other procedures like prediction and updating operation similar to that of DGKD, but the difference is the metric employed in their systems. Experimental comparison between the two technique shows that PDGKD achieves better result than DGKD. However, the limitation of the system arose when kidnap robot happens over a long period of time.

In the technique of (Tan et al., 2015), simultaneous localization and mapping without linearization was proposed to overcome the issue of linearization and inconsistency caused by approximation limiting the effectiveness of EKF-SLAM. This was achieved by combining both linear time varying Kalman filter and contraction tools on navigation problem with virtual measurements. Given a robot with LTV Kalman filter SLAM using a virtual measurement in local coordinates, the EKF is the basis of LTVK and it would start with available non-linear measurement given in equation (26)

$$
\theta=\arctan \left(\frac{x_{a}}{x_{b}}\right) \text { and/or } r=\sqrt{x_{a}^{2}+x_{b}^{2}}
$$

where $\theta$ represent the measure azimuth angle of the robot, $\left(x_{a}, x_{b}\right)$ represent location of landmark and $r$ represent range measurement between robot and landmark. Afterwards the estimated Jacobian is employed to linearize this measurement to a locally stable observer. Expression of the relation in Cartesian coordinate is given in equation (27).

$$
h \mathrm{x}=0 \quad \text { and } / \text { or } \quad h * \mathrm{x}=r
$$

But in 2D and 3D scenarios, expression is given in equations (28) and (29)

$$
\begin{gathered}
h=(\cos \theta,-\sin \theta) \quad \text { and } / \text { or } \\
h=\left(\begin{array}{ccc}
\cos \theta & -\sin \theta & 0 \\
-\sin \phi \sin \theta & -\sin \phi \cos \theta & \cos \phi
\end{array}\right) \text { and /or } \quad h^{*}=(\sin \theta, \cos \theta)
\end{gathered}
$$

Given a linearize equation in local coordinate, the azimuth model representation for actual location of a landmark in $2 \mathrm{D}$ and $3 \mathrm{D}$ is expressed in equation (30) and (31)

$$
\begin{aligned}
& \mathrm{x}=\left(x_{1}, x_{2}\right)^{T}=(r \sin \theta, r \cos \theta)^{T} \\
& \mathrm{x}=\left(x_{1}, x_{2}\right)^{T}=(r \cos \phi \sin \theta, r \cos \phi \cos \theta, r \sin \phi)^{T}
\end{aligned}
$$

where $h / h^{*}$ represent state independent measurement vector, $\phi$ represent the actual location of a landmark.

In LTVK, measurement and observation between $\theta$ and $r$ are ignored while feedback on tangential and Cartesian position errors between estimated and true landmark position $(\mathrm{x})$ are taken into consideration. Therefore, the linear observation given above is substituted by virtual measurement, expression in Cartesian coordinate is given in equation (32) 


$$
y=H x+v(t)
$$

where $y$ represent observation/measurement vector which contains virtual/actual measurement, $H$ represent observation model matrix that include state-independent measurement vectors and $v(t)$ represent zero-mean white noise. If further exploited can be employed to attain a globally stable observer design with no set back error caused by linearization operation. However, this achievement is at a price of high computational cost and in their future work, they plan to reduce the computational workload by using the technique suggested by $(\mathrm{Mu}, 2013)$. The technique proposed the use of landmark with more provided information for feature selection to assist in reducing computational workload.

In the work of (Jia et al., 2016) vision-based technique using a monocular camera was proposed to initiate SLAM. The algorithm employed in their technique is known as Parallel, Tracking and Mapping (PTAM). In PTAM, procedures are split into two level task operations in parallel. In the tracking level, monocular camera fixed on mobile robot is used to capture images from the environment. Ground feature-based pose estimation algorithm was proposed to detect ground features. But to achieve a more accurate robot pose, a weighted projection error-based energy function expressed in (33) was used to achieve this task.

$$
\min _{\mathrm{x}} \sum w\left(r_{p}\right)\left\|r_{p}(\mathrm{x})\right\|
$$

where $w$ represent the tukey biweight function for the homography-based projection error $\left(r_{p}\right)$.

Given the accurate robot localization attained by the expression in (33), matched features triangulated was used to generate an initial map. Afterwards is the second level task known as mapping thread, during this task, the initialized map is queried for incorporating new key features and these is achieved by employing the use of epipolar searching procedure. The new matched features are selected for acceptance by searching for candidate region around the epipolar with minimal differences of Zero-Mean Summed Squared Differences (ZMSSD), while candidate region with higher differences in ZMSSD compared to the threshold will be rejected for re-mapping. Expression is given in equation (34)

$$
s\left(u_{j}^{i}\right)=\left\{\begin{array}{l}
r_{j} \leq \delta_{i} \text { accept } \\
r_{j}>\delta_{i} \text { reject }
\end{array}\right.
$$

where

$$
r_{j}=\pi\left(H_{i} v_{j}^{i}\right)-u_{j}^{i},
$$

$H_{i}$ represent the homography estimated by RANSAC algorithm, $v_{j}^{i}$ signifies the reference feature of $u_{j}^{i}, r_{j}$ represent the projection error and $\delta_{i}$ represent the threshold.

After classification using equation (34), new map points generated are in-cooperated into the map points to improve the accuracy of the system. Indoor experimental performance carried out shows tremendous achievement towards accuracy, but from the future work presented in their study, they intend to improve the performance of their technique to cope with mapping in various illumination scenarios. 
The work presented in the study of (Agarwal and Burgard, 2015) is a graph based simultaneous localization and mapping. The concept of graph-based SLAM relies on representing the nodes present in the graph by each pose attained by the robot. In real world scenario, these nodes can be used to signify features extracted from images captured by camera sensors or laser point cloud. Nodes can also be employed to signify physical landmark of object like trees, cars etc. Edges present in the graph is signified by a factor connecting two nodes. These factors represent the bearing measurement of features. Given a robot navigating in an unknown environment using graph-based SLAM, the first problem to address is creating a graph. This can be attained by identifying the nodes and the factors connecting these nodes based on the data generated from the sensors, this computation is known as front-end. The second problem to address is the nodes configuration that provides best explanation for the factors. These steps assist to compute a maximum likelihood map and these computations is known as back end. Thus, the back ends aim to find configuration of nodes that minimize error created by the factors from the front-end operation. If $x=\left(x_{1}, \ldots \ldots ., x_{n}\right)^{T}$ represent a state vector and $x_{i}$ signifies pose of node $i$ which can also represent a robot or landmark position. The error function $\left(e_{i j}(x)\right)$ description for a single factor between $i$ and $j$ represent the different between $Z_{i j}$ and $\hat{Z}\left(x_{i}, x_{j}\right)$. Expression is given in equation (35).

$$
e_{i j}(x)=\hat{Z}\left(x_{i}, x_{j}\right)-Z_{i j}
$$

where $\hat{Z}\left(x_{i}, x_{j}\right)$ signifies the expected measurement given in the current state, $Z_{i j}$ represent the observed measurement, $i$ and $j$ represent the graph nodes. The re-projection error of the observed landmark must be minimized for accuracy purpose. Thus, the minimization error can be expressed in equation (36)

$$
\mathrm{x}^{*}=\underset{x}{\arg \min } \sum_{i j} e_{i j}(x)^{T} \sum_{i j}^{-1} e_{i j}(x)
$$

where $\sum_{i j}^{-1}$ represent information matrix related to the factors that exist between two pose $x_{i}$ and $x_{j}, \sum_{i j}$ signifies the covariance matrix and $\mathrm{x}^{*}$ represent the optimal configuration of nodes with limited error induced from the factors of front end operation. However, the effectiveness of graph based technique towards SLAM has attracted researcher like (Agarwal and Burgard, 2015). In their enhanced graph-based SLAM, experimental result shows tremendous success towards SLAM problem, but at an expense of high computational cost due to an increase in computational requirement at the matrix factorization stage. Furthermore, their graph-based SLAM couldn't cope with dynamic environment and in their future work, they will be improving the SLAM technique towards tracking of dynamic object.

The technique proposed in the work of (Li-Chee-Ming and Armenakis, 2016) is to address the problem of SLAM required in the combination of Visual Serving Platform (VISP) and RGBD-SLAM. The VISP is a common used open source tool for tracking relative pose between the camera and the model of an object. The VISP is capable of carrying out the task of extracting the features important to address the problem of SLAM. It entails the moving edge (ME) algorithm proposed by (Bouthemy, 1989) for feature extraction 
by matching the feature point in an image and projected model while the current pose of the camera is estimated using a non-linear optimization method known as virtual visual servony. However, relying on VISP alone might not be sufficient because issue arose when tracking is missing, there will be need for re-initialization. This issue can occur as a result of lack of model features in the sequence of image captured by the camera, rapid camera motion can also be a contributor to loss of tracking. Therefore, improving the tracking becomes very important and RGB-D SLAM was proposed concurrently to provide the VISP the missing tracking. The RGB-D SLAM is a graph-based approach similar to the one proposed in the work of (Agarwal and Burgard, 2015) which involves the frontend and backend operation. Given in Figure 2 is a model representing the integration workflow between VISP and RGB-D SLAM

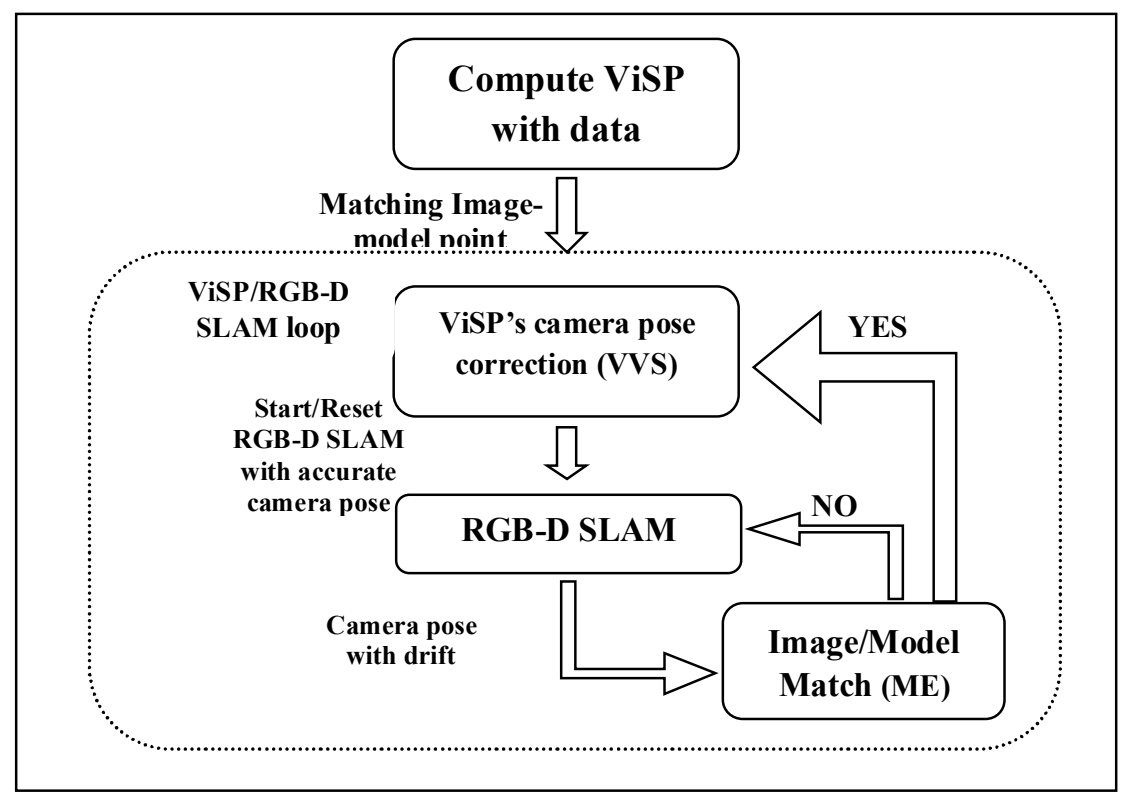

Figure 2: RGB-D SLAM /ViSP integration workflow (Li-Chee-Ming and Armenakis, 2016)

The collaboration was successful and experiment performance carried out on the second floor of York University, Bergeron Centre for Engineering and Excellence shows tremendous improvement towards recovery of lost tracking but at the expense of high computational cost. The experiment further reveal that the computational issue didn't happen at the VISP operation rather it happened at the RGB-DSLAM running without resetting for an extended period of time, as a result of processing simultaneously huge size of data. However, in the work of (Agarwal and Burgard, 2015), they also proposed RGB-D graphbased SLAM technique and experience the issue of computational cost. In conclusion, could it be that computational issue is a general problem associated with RGB-D SLAM technique?

(Irie et al., 2012) proposed to address the problem of SLAM for outdoor navigation taking into consideration drastic illumination changes which happen in most environment. In this technique, stereo camera capable of obtaining 3-D range data was employed to capture data from the environment. Afterwards, 2-D grid map that is not much affected by illumination condition is generated. Given the 2-D grid map, occupancy information and salient line segment can be extracted perfectly. The particle filter is employed to extract the robot pose while edge pint based stereo SLAM was used to obtain robot ego 
motion and the occupancy information simultaneously. This extracted information is used to address the SLAM problem. There are other important procedures carried out to develop their technique, the model in Figure 3 provides full description of the proposed SLAM technique for mobile robot navigation in outdoor environment.

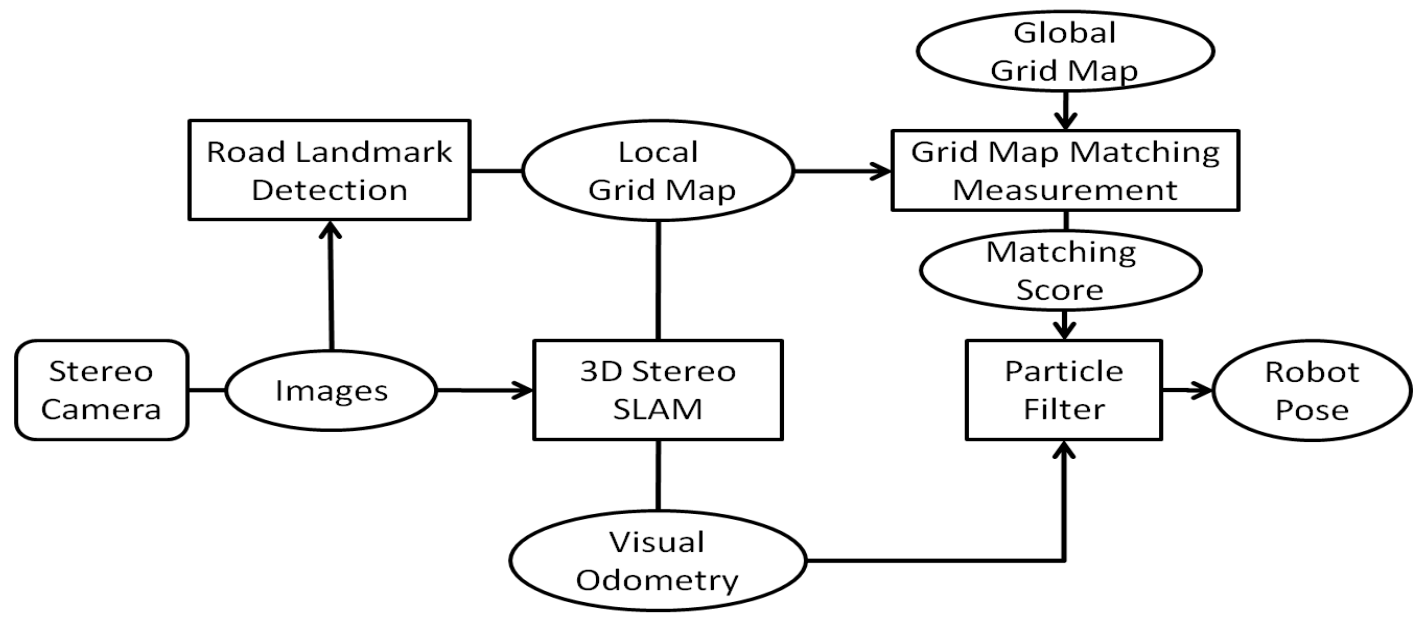

Figure 3: represent the stereo SLAM technique for mobile robot in outdoor environment (Irie et al., 2012)

The proposed model was successfully implemented and experimental performance of their visual odometry recovered from error and performed very well under various illumination situations. However, the technique failed under extremely adverse illumination condition such as when direct sunlight covers large part of the image and giving this condition, limited edge point are extracted for detection which resulted to huge error in motion estimation and inability to recover from kidnap robot.

In the research work of (Oh et al., 2015), they proposed to develop a Simultaneous Localization and Mapping (SLAM) technique using a monocular camera and a 2-D laser scanner sensor. The two sensors were encouraged because of environment with ambiguity such as long corridor, SLAM algorithm working with laser scanners might not be able to estimate correctly the robot position. In resolving this problem, monocular camera was introduced into their system to collect data from the environment. Figure 4 illustrate how the proposed SLAM technique make use of the two sensors. 


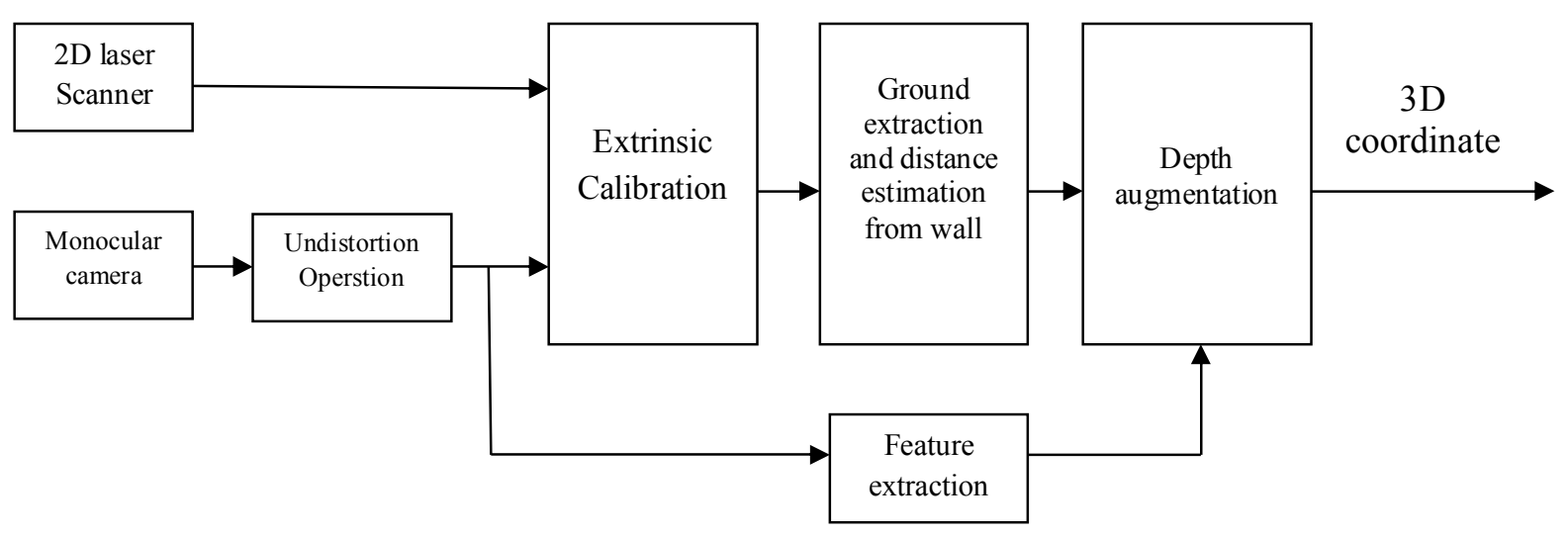

Figure 4: the proposed monocular camera and laser scanners sensors SLAM technique (Oh et al., 2015)

At the monocular camera stage, graph structure-based technique and hybrid method which allows the SLAM technique to estimate the robot pose in ambiguity environment was proposed where laser scanner fails. In a graph-based technique, the SLAM problem using a conditional probability is expressed in (37).

$$
p(x \mid z) \alpha \prod_{i} p\left(z_{i} \mid x\right)
$$

where $x$ signifies the robot pose, $z=\left(z_{1}, \ldots \ldots ., z_{n}\right), z_{i}$ signifies measurement of a sensor at $i^{\text {th }}$ step while $p\left(z_{i} \mid x\right)$ represent a potential function.

Given this technique, experimental comparison was carried out with a conventional G-mapping approach and results revealed that their system performs better than the G-mapping approach. However, as mentioned in the section of their results, the algorithm proposed has a small computational burden because more image processing is executed when a node is added. This was also supported to be true because in the work of (Deming and Perlovsky, 2006), it was stated that data association from multiple sensors can cause the computational complexity of systems to be prohibitively high with an effect that can lead to system failure.

The study of (Clipp et al., 2009) proposed a Simultaneous Localization and Mapping (SLAM) technique using a stereo camera as the source of retrieving information from the environment. In their technique, they took into consideration the issue of computational cost and propose the use of Kanade-Lucas-Tomasi (KLT) feature tracking because of its advantage of high-speed in processing and robustness to features that are repetitive in nature; this technique was combined with a wide baseline feature which helps the system to improve its robustness to repetitive features and allows it to recognize previously visited areas in the environment. Experimental performance of the system is impressive. However, as stated in their conclusion, an item on the desk in front of the windows appeared blank due to the presence of high intensity of bright sunlight and also, the system was unable to recognize the area that has already been mapped due to movement of objects in the same area and this is as a result of low dynamic range of the system. Thus, these two problems when encountered which makes their system to fail. In the future, they plan to address this issue by extracting features in 3D scene just as it was proposed in the work of (Changchang et al., 2008). The idea is to look for ways of combining 3D geometry and sparse feature 
detection with the aim of using immovable features in the image such as floor, wall and ceiling to relocalize itself regardless of dynamic changes happening in the environment. Successful implementation of this idea will allow the system to overcome the issue of dynamic environment and illumination variances

\section{CHALLENGES, OPEN ISSUE AND RESEARCH DIRECTION}

The SLAM domain has experienced a lot of research and various effective methods have been recommended. Thus, there is no general technique of handling the complex problems various researchers experience during the implementation of their SLAM techniques, but there has been improvement towards addressing some of these issues. Take for instance, the issue of illumination variance which happens as a result of object obstructing light generated from a source has not been fully addressed, but to some extent in static environment, the effect has been minimized in the work of (Agunbiade et al., 2014, Sujan et al., 2006). In dynamic environment, the effect of illumination variation becomes difficult to address. Given a condition where static object cast dynamic illumination, localization becomes complicated. It's even more problematic and challenging to estimate localization if a dynamic object is casting a dynamic illumination (Le Cras et al., 2013). A different problem that has not been fully resolved is the issue of kidnap problem, although it has received a lot of attention from researchers with improvement on it. The kidnapping problem occurs when the robot moves from one position to another without having any information about its new position (Guyonneau et al., 2012) and this can happen as a result of failing sensors or increase in measurement noise (Negenborn et al., 2003). Meanwhile, when a robot is kidnapped in an environment, algorithms selected to solve this problem must be able to carry out these objectives: ability to perform pose estimation, detect kidnap problem and global localization (Guyonneau et al., 2012). However, in the literature, the initializing localization technique has been positive to kidnap robot (Se et al., 2001). In this technique, an extensive search of the current observation over the reference map (pre-mapped environment) will be carried out to find the robot position in relation to this map. This will assist the robot to start up again at the last stop position since the last position has already been mapped in the previous map. But in the situation where the current observation previously map changed as a result of dynamic object present in the environment. This becomes challenging to address because it will be unable to locate its current observation from the referenced map and relocalization becomes impossible which might lead to robot lost without recovery (Se et al., 2001). However, the dynamic environment which contribute towards complicating other problem is also an open problem that researchers are still studying till date. Achievement in this research area depend on how dynamic the environment is, the more dynamic the environment, the more difficult and challenging to address (Walcott-Bryant et al., 2012). Lastly, conducting a review in SLAM is incomplete without mentioning the computational cost as related to real-time. This is widely complained by previous researchers working on SLAM (Agarwal and Burgard, 2015, Castellanos et al., 2001, Oh et al., 2015). In literature, some researchers have proposed wide range of algorithm with mathematic technique to attain impressive result towards computational cost (Dellaert et al., 2010, Kaess et al., 2008, Konolige et al., 2010), but these algorithms have their own advantages and disadvantage (Dissanayake et al., 2011). Take for instance the FAST-SLAM commonly used by researchers (Montemerlo and Thrun, 2003, Qiu et al., 2012) trying to limit computational cost relies heavily on particles. The accuracy of FAST-SLAM is dependent on the number of particles which can also increases the computational complexity (Wurm et al., 2003). Some researchers also proposed to improve the accuracy by introducing more algorithm to enhance the SLAM system performance, but with an effect that increases the computational complexity (Li-Chee-Ming and Armenakis, 2016, Yinka et al., 2014). In both situation it becomes a tradeoff between 
accuracy and SLAM runtime. Therefore, researchers are left to decide whether to reduce the accuracy of the SLAM system to decrease it computational cost or vice versa. In future, the greatest accomplishment is to attain a globally optimal solution that will address all SLAM problems. In attaining this goal, more investigation is still necessary to be carried out for proper understanding of some problem. Given a robot in real life scenario, it should be able to localize and mapped in various environment condition like indoor, out-door and under water environment. In any environment, object is often present, some objects are static while some are dynamic in nature. Therefore, SLAM technique must be capable of modeling the environment at object level, most especially in human predominant environment. Furthermore, Noises such as environmental noises (shadow and light intensity), sensors noises, gaussian noise etc all need to be eliminated to attain a SLAM technique with maximum accuracy. Even though we've discussed some issues that needs to be resolved to present the future SLAM. However, all problems need to be accomplished in real-time.

\section{THE PROPOSED NOVEL SLAM TECHNIQUE}

Over the years, researchers have proposed various SLAM technique, but they were confronted with one problem or the other. In this review, some of the problems discussed are as follows: Illumination variation, kidnap robot, dynamic environment and high computational cost. Thus, all these problems if not fully addressed can be minimize towards improving the SLAM performance. In the proposed SLAM technique, filters could be employed to minimize the effect of illumination variation of shadow and light intensity. In literature Normalize Difference Index is a common algorithm towards minimizing the effect of shadow (Agunbiade et al., 2014) while dark channel prior and OTSU thresholding algorithms are used to minimize light intensity (Agunbiade et al., 2014). These filters could be operating in parallel since we are taking into consideration the issue of high computational cost. The kidnap problem which contribute to localization failure can also be addressed by introducing into the proposed SLAM the scan to match algorithm to search for the reference map of the current observation for re-localization of the robot (Se et al., 2001). The dynamic issue can be minimized by introducing the enhanced fuzzy clustering technique for keeping track of multiple dynamic object in the environment (Walcott-Bryant et al., 2012). The SLAM algorithm is most likely to be particle based, because of it satisfactory result and acceptable performance towards computational cost. However, with the proposed novel SLAM technique possessing all these characteristics, it will offer better performance than any of the technique discussed in this review.

\section{Conclusion}

In this study, review on various SLAM algorithms was conducted, to understand how SLAM problem are being addressed, and the trend of problem encountered by researchers. SLAM has attracted many researchers because it supports the possibilities of concurrent execution of mapping and localization process. These become a great accomplishment in solving the problem of mobile robot autonomously achieving its goal without being controlled by anyone. More so, improving on this research area will require addressing problems associated with current SLAM. Therefore, review was conducted on recent and foundation SLAMf in order to assist us to discover persistence and recent problem associated to current SLAM techniques. However, the observation from this study suggested that new researchers must focus their attention on the issue of high computational cost as related to processing time, this is a major problem mostly complained in SLAM. In addition, other problems such as illumination variance (light 
intensity and shadow), kidnap robot and dynamic environment are persistence problem mentioned by the researchers in their studies. Figure 5 shows the overall impact of these SLAM problems as related to this research.

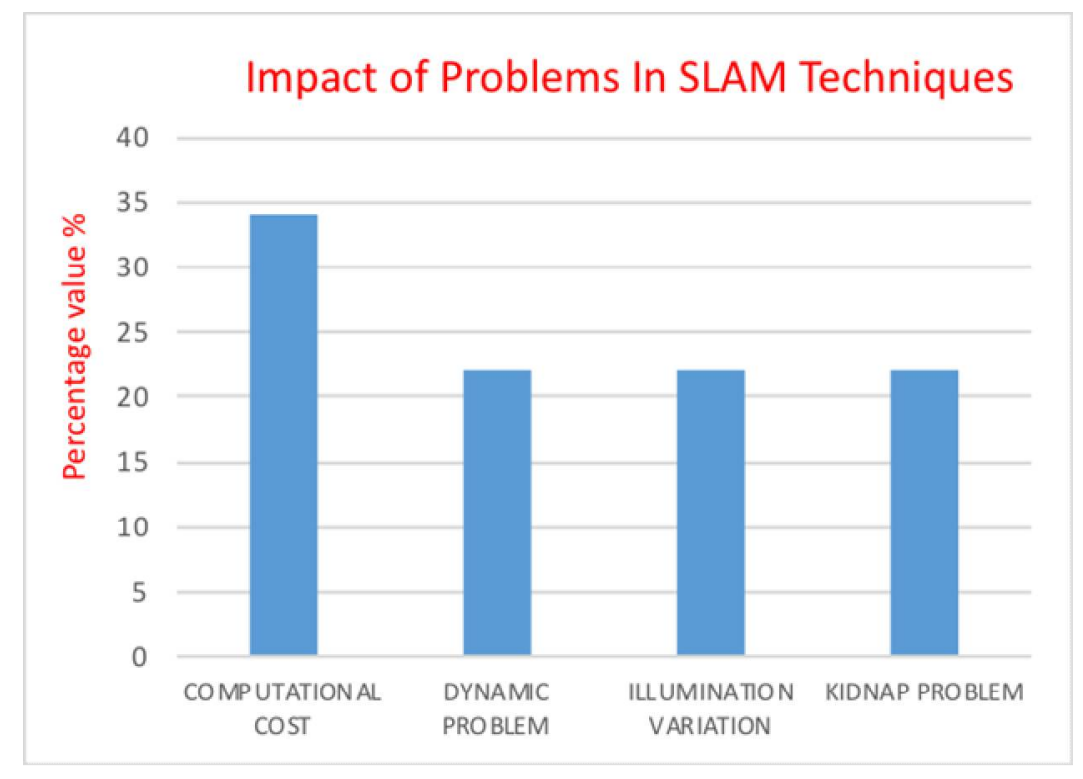

Figure 5: The overall impact of the SLAM problems

Furthermore, the advantages and limitations of sensors as related to SLAM problem were also discussed. In our future work, we will be implementing the proposed SLAM technique and successful execution will improve the performance of autonomous robot in path planning, exploration and mission planning. 


\section{References}

1. AGARWAL, P. \& BURGARD, W. 2015. Robust graph-based localization and mapping, PhD thesis,. University of Freiburg, Germany, 1-155.

2. AGHA-MOHAMMADI, A.-A., AGARWAL, S., CHAKRAVORTY, S. \& AMATO, N. M. 2015. Simultaneous Localization and Planning for Physical Mobile Robots via Enabling Dynamic Replanning in Belief Space. Robotics: Systems and Control, abs/1510.07380, 1-21.

3. AGUNBIADE, O. Y., ZUVA, T., JOHNSON, A. O. \& ZUVA, K. 2014. Enhancement performance of road recognition system of autonomous robots in shadow scenario. Signal \& Image Processing : An International Journal (SIPIJ), abs/1401.2051, 1-10.

4. BOUTHEMY, P. 1989. A maximum likelihood framework for determining moving edges. IEEE Transactions on Pattern Analysis and Machine Intelligence, 11, 499-511.

5. CASTELLANOS, J. A., NEIRA, J. \& TARDOS, J. D. 2001. Multisensor fusion for simultaneous localization and map building. IEEE Transactions on Robotics and Automation, 17, 908-914.

6. CHANGCHANG, W., CLIPP, B., XIAOWEI, L., FRAHM, J. M. \& POLLEFEYS, M. 3D model matching with Viewpoint-Invariant Patches (VIP). 2008 IEEE Conference on Computer Vision and Pattern Recognition, 23-28 June 2008 2008. 1-8.

7. CHEN, Y. 2013. Algorithm for Simultaneous Localization and Mapping. 1-15.

8. CLIPP, B., ZACH, C., LIM, J., FRAHM, J.-M. \& POLLEFEYS, M. 2009. Adaptive, Real-Time Visual Simultaneous Localization and Mapping. Department of computer Science, University of Noorth Caroline, 1-8.

9. DELlAERT, F., CARLSON, J., ILA, V., NI, K. \& THORPE, C. E. Subgraph-preconditioned conjugate gradients for large scale SLAM. 2010 IEEE/RSJ International Conference on Intelligent Robots and Systems, 18-22 Oct. 2010 2010. 2566-2571.

10. DEMING, R. \& PERLOVSKY, L. Concurrent Detection and Tracking using Multiple, Flying, Sensors. Fourth IEEE Workshop on Sensor Array and Multichannel Processing, 2006., 12-14 July 2006 2006. 505-509.

11. DISSANAYAKE, G., HUANG, S., WANG, Z. \& RANASINGHE, R. A review of recent developments in Simultaneous Localization and Mapping. 2011 6th International Conference on Industrial and Information Systems, 16-19 Aug. $20112011.477-482$.

12. FUENTES-PACHECO, J., RUIZ-ASCENCIO, J. \& RENDÓN-MANCHA, J. M. 2015. Visual simultaneous localization and mapping: a survey. Artificial Intelligence Review, 43, 55-81.

13. GUYONNEAU, R., LAGRANGE, S., HARDOUN, L. \& LUCIDARME, P. 2012. The Kidnapping Problem of Mobile Robots: A Set Membership Approach. 7th National Conference on Control Architectures of Robots.

14. HADJI, S. E., KAZI, S. \& HING, T. H. 2014. A Review: Simultaneous Localization and Mapping Algorithms for Mobile Robot. $1^{\text {st }}$ International Conference of Recent in Informtion and Communication Technologies, 83-92.

15. IRIE, K., YOSHIDA, T. \& TOMONO, M. 2012. Outdoor Localization Using Stereo Vision Under Various Illumination Conditions. Advanced Robotics, 26, 327-348.

16. JIA, S., WANG, K. \& LI, X. 2016. Mobile Robot Simultaneous Localization and Mapping Based on a Monocular Camera. J. Robot., 2016, 2.

17. KAESS, M., RANGANATHAN, A. \& DELLAERT, F. 2008. iSAM: Incremental Smoothing and Mapping. IEEE Transactions on Robotics, 24, 1365-1378.

18. KHAIRUDDIN, A. R., TALIB, M. S. \& HARON, H. Review on simultaneous localization and mapping (SLAM). 2015 IEEE International Conference on Control System, Computing and Engineering (ICCSCE), 27-29 Nov. 2015 2015. 85-90. 
19. KONOLIGE, K., GRISETTI, G., KÜMMERLE, R., BURGARD, W., LIMKETKAI, B. \& VINCENT, R. Efficient Sparse Pose Adjustment for 2D mapping. 2010 IEEE/RSJ International Conference on Intelligent Robots and Systems, 18-22 Oct. 2010 2010. 22-29.

20. LE CRAS, J., PAXMAN, J. \& SARACIK, B. 2013. Improving Robustness of Vision Based Localization Under Dynamic Illumination. In: SEN GUPTA, G., BAILEY, D., DEMIDENKO, S. \& CARNEGIE, D. (eds.) Recent Advances in Robotics and Automation. Berlin, Heidelberg: Springer Berlin Heidelberg.

21. LI-CHEE-MING, J. \& ARMENAKIS, C. 2016. Augmenting ViSP's 3D Model-Based Tracker with RGB-D SLAM for 3D Pose Estimation in Indoor Environments. Int. Arch. Photogramm. Remote Sens. Spatial Inf. Sci., XLI-B1, 925-932.

22. MONTEMERLO, M. \& THRUN, S. Simultaneous localization and mapping with unknown data association using FastSLAM. 2003 IEEE International Conference on Robotics and Automation (Cat. No.03CH37422), 14-19 Sept. 2003 2003. 1985-1991 vol.2.

23. MU, B. 2013. Value of Information based distributed inference and planning. PhD Thesis, Massachusetts Institute of Technology.

24. NEGENBORN, R., JOHANSEN, P. P. \& WIERING, M. 2003. Robot Localization and Kalman Filter. Institute of Information and Computing Sciences.

25. OH, T., LEE, D., KIM, H. \& MYUNG, H. 2015. Graph Structure-Based Simultaneous Localization and Mapping Using a Hybrid Method of 2D Laser Scan and Monocular Camera Image in Environments with Laser Scan Ambiguity. Sensors, 15, 15830.

26. QIU, C., ZHU, X. \& ZHAO, X. Vision-based unscented FastSLAM for mobile robot. Proceedings of the 10th World Congress on Intelligent Control and Automation, 6-8 July 2012 2012.3758-3763.

27. SE, S., LOWE, D. \& LITTLE, J. Vision-based mobile robot localization and mapping using scale-invariant features. Proceedings 2001 ICRA. IEEE International Conference on Robotics and Automation (Cat. No.01CH37164), 2001 2001.2051-2058 vol.2.

28. SUJAN, V. A., MEGGiOlARO, M. A. \& BELO, F. A. W. 2006. A new technique in mobile robot simultaneous localization and mapping. Sba: Controle \& Automação Sociedade Brasileira de Automatica, 17, 189-204.

29. TAN, F., LOHMILLER, W. \& SLOTINE, J.-J. E. 2015. Simultaneous Localization And Mapping Without Linearization. Massachusetts Insitute of Technology, abs/1512.08829, 1-50.

30. TIAN, Y. \& MA, S. 2016. Probabilistic double guarantee kidnapping detection in SLAM. Robotics and Biomimetics, 3, 20.

31. WALCOTT-BRYANT, A., KAESS, M., JOHANNSSON, H. \& LEONARD, J. J. Dynamic pose graph SLAM: Long-term mapping in low dynamic environments. 2012 IEEE/RSJ International Conference on Intelligent Robots and Systems, 7-12 Oct. 2012 2012. 1871-1878.

32. WURM, K. M., STACHNISS, C., GRISETTI, G. \& BURGARD, W. 2003. Improved Simultaneous Localization and Mapping using a Dual Representation of the Environment. Department of Computer Science, University of Freiburg, Germany, 1-10.

33. YINKA, A. O., NGWIRA, S. M., TRANOS, Z. \& SENGAR, P. S. Performance of drivable path detection system of autonomous robots in rain and snow scenario. 2014 International Conference on Signal Processing and Integrated Networks (SPIN), 20-21 Feb. 2014 2014. 679-684.

34. ZEHANG, S., BEBIS, G. \& MILLER, R. 2006. On-road vehicle detection: a review. IEEE Transactions on Pattern Analysis and Machine Intelligence, 28, 694-711. 